EUROPEAN JOURNAL OF ENVIRONMENT
European Journal of Environment and Public Health, 2017, 1(2), 10

ISSN: $2468-1997$

\title{
An Analysis of Depression and Diabetes amongst African American Women
}

\author{
Jonathan Cooke 1 , Henry O'Lawrence ${ }^{1 *}$ \\ ${ }^{1}$ Health Care Administration Department, College of Health and Human Services, California State University, Long Beach, \\ $C A$, USA
}

*Corresponding Author: Henry.OLawrence@csulb.edu

Citation: Cooke, J. and O'Lawrence, H. (2017). An Analysis of Depression and Diabetes amongst African American Women. European Joumal of Emvironment and Public Health, 1(2), 10. https://doi.org/10.20897/ejeph/81157

Published: December 30, 2017

\begin{abstract}
This study determined the level of depression and the rate of diabetes among the adult African American female population. By identifying the population early, one can save millions of dollars, improve health outcomes, and treat depression that typically interferes with diabetes management. The study used secondary data from the 2011-2012 California Health Interview Survey. The 2011-2012 CHIS surveyed 42,935 adults and of those surveyed 1,403 respondents identified themselves as African Americans. Chisquare analysis was used to determine the strength of the association between diabetes in African American women with depression and exercise. The results of the statistical tests show that there was an association between the independent variables suggesting a statistically significant relationship in African American women. A major limitation of the study was the small number of African Americans interviewed in the survey. A larger sample size at the national level is recommended to more closely scrutinize the directional nature of the relationship between diabetes and depression in African American women.
\end{abstract}

Keywords: depression, diabetes, African Americans, health care administration

\section{INTRODUCTION}

Diabetes burden has been much larger in minority populations than in the white population. For example, about $13 \%$ of non-Hispanic blacks and a little over 13\% of Mexican Americans have diabetes as contrasted to about 7\% of non-Hispanic whites (American Diabetes Association "Diabetes Statistics", 2013a). African Americans have a long history of diabetes in the United States compared to what has been the historical majority of whites. Diabetes incidence rates have increased significantly in the U.S. population. Minority groups have disproportionately shared the highest rates. Minority groups have included Latinos, Native Americans, and African Americans. The highest rates of diabetes in African Americans have occurred in rural South Eastern African Americans (Cummings et al., 2013).

Depression, usually considered a comorbid condition, adds to the burden of diabetes. Diabetes alone presents multiple health problems but the addition or combined effect of depression results in devastating effects. Too often, general practitioners fail to use a well-respected screening tool like the PHQ 2/9 inventory for depression. Motivational Interviewing might provide one way for physicians to clear obstacles like cultural competence (Miller et al., 2010). Depression leads to lack of exercise and lack of medication adherence. Furthermore, depression exacerbates diabetes symptoms, and compounds negative perceptions about the health system.

African Americans continue to have a disproportionate share of diabetes despite all the advances made in modern medicine. The previous studies mentioned have described many other potential causes such as socioeconomic status, everyday discrimination, diabetes related distress, depression, cultural differences, and 
distrust of the healthcare system. This study is being performed to bring awareness to diabetes, health consequences of diabetes, and the disproportionate burden shared by African American women. Diabetes is a worldwide epidemic and minority groups in the United States disproportionately share in the prevalence and incidence rates of diabetes (Black, 2002). To give an idea of the scale, Wild et al. (2004) project into the year 2030 and estimate that the rise of worldwide diabetes rates to expand from 171 million in 2000 to 366 million in 2030. Depression exacerbates the symptoms of diabetes through interfering with medication adherence and other selfcare behaviors needed to properly treat diabetes (Katon et al., 2013).

In absolute numbers, non-Hispanic whites exceed African Americans in diagnoses of diabetes whereas African Americans exceed whites in diagnoses of diabetes by $8.5 \%$ for adults aged 20 years or older (Centers for Disease Control, 2011). As further evidence of African Americans disproportionately affected by diabetes, $18.7 \%$ of all African Americans aged 20 years and older have diabetes (American Diabetes Association, 2013a). Compared to non-Hispanic Whites, African Americans are more than 1.8 times likely to have diabetes (American Diabetes Association "Diabetes Statistics", 2013a). African Americans have nearly 50\% greater likelihood of developing diabetic retinopathy when compared to non-Hispanic whites. Diabetes has life-threatening health consequences if left untreated or unmanaged; and also, blindness is one example of a complication that may develop if diabetes goes untreated (American Diabetes Association, 2013b).

According to Murrock et al. (2013) four main themes emerge in managing the dietary challenges of African American women. The researchers conducted a qualitative focus group approach. The significance of the study stems from 2.7 million African American adults living with diabetes, and about a quarter of whom are women aged 50 or older. Also, African American women are more likely than African American men to develop health problems like obesity and high blood pressure. One limitation of the study was the phenomenological approach used to explore meaning and describe human experiences. Additionally, the study focused only on African American women with Type 2 diabetes and the exclusion criteria removed anyone with severe mental illness; Murrock et al. (2013) report future studies should include larger populations and thus be more generalizable than their own. One assumption made during the study was the participants were answering questions honestly as opposed to provide socially acceptable answers; with such a small group of people being interviewed, lack of bias is hard to discern. The two main themes which emerged relating to the dependent variable (glycemic control) were (a) the frequent struggles and difficulties in managing diabetes and (b) lack of individualized guidance (c) social support (d) misinformation in diabetes knowledge. To contribute to the literature, the authors conducted a study that emphasized the need to educate and manage the dietary skills amongst African American women who have trouble controlling glycemic levels.

Cummings et al. (2013) argued that most African Americans in the rural south experience less weight loss and glycemic control than whites in diabetes management programs. In this study, rural African Americans in the South received a randomized prospective intervention approach to help manage Type 2 diabetes. The population under study was African American women adults with a history of Type 2 diabetes and $\mathrm{HbA}_{1 c}$ levels indicating poor glycemic control. Community health workers (CHW) were employed to help the patient select their own goals and to make small changes. Any one small change would not significantly alter numbers to desired ones but rather the intervention was designed to accumulate small, sustainable behavior changes.

With enough accumulated behavior changes, a significant impact could be made on the health of an individual. After excluding people not meeting eligibility criteria, a study sample size of 200 was formed. Many participants were categorized as low SES (41.5\% reporting less than $\$ 10,000$ per year income) and reported difficulty in following any kind of meal plan or any kind of exercise regimen (71.9\% and 75.3\%). Results from the study showed that interventions should not solely focus on educational aspects of disease management but on coping skills and behaviors promoting mental health that may be necessary for treatment outcomes. Findings indicated a greater chance of changing behavior using the EMPOWER-CHW than educational materials alone. The authors created a replicable study that accounted for available resources, cultural competence, participant SES, and incremental, effective behavior changes (Cummings et al, 2013).

\section{The Data and Statistical Analysis}

Unlike previous versions of California Health Interview Survey (CHIS), in 2011 CHIS changed to a survey method of continuously collecting data during each two year cycle. In the CHIS 2011-2012 survey, adult questionnaire version 10.1 was used to collect data from participants 18 years older from various ethnic groups and parts of California. Although more than three agencies were listed in the beginning, the three main collaborating agencies were UCLA-CHPR, California Department of Health Care Services, and the California Department of Public Health.

To perform the survey, CHIS randomly selects one adult to interview for each participating household. Acting on behalf of a child, an adult with the greatest knowledge about the child (age 0-11) was interviewed and for adolescents (age 12-17) interviewed if granted permission by parents. For analysis, sections of the CHIS 2011-2012 
Table 1. CHIS 2011-2012 Racial/Ethnic Groups Frequencies

\begin{tabular}{|c|c|c|}
\hline Total Sample Size & Adult (Age 18+) & $N=42,935$ \\
\hline & African American & 2,102 \\
\hline & American Indian/Alaska Native & 790 \\
\hline & Asian & 4,302 \\
\hline Race * & Pacific Islander & 82 \\
\hline & White & 30,110 \\
\hline & Other single race & 4,585 \\
\hline & Two or More Races & 964 \\
\hline
\end{tabular}

*2000 Census definition of race

Table 2. Demographics of Adult Survey Participants $(N=42,935)$

\begin{tabular}{|c|c|c|c|}
\hline \multicolumn{2}{|c|}{ Characteristics } & Participants (N) & $\%$ \\
\hline \multirow{2}{*}{ Gender } & Male & 17,848 & 41.6 \\
\hline & Female & 25,087 & 58.4 \\
\hline Age & $18+$ & 42,935 & 100 \\
\hline \multirow{4}{*}{ Marital Status } & Married & 21,361 & 49.8 \\
\hline & Living with partner & 2,260 & 5.3 \\
\hline & Widow/Separated/Divorced & 11,848 & 27.6 \\
\hline & Never married & 7,466 & 17.4 \\
\hline \multirow{12}{*}{ Self-reported Race } & African American & 2,318 & 5.4 \\
\hline & American Indian & 1,408 & 3.3 \\
\hline & Asian & 4,526 & 10.5 \\
\hline & Chinese & 1,200 & 2.8 \\
\hline & Filipino & 601 & 1.4 \\
\hline & Korean & 739 & 1.7 \\
\hline & Latino/Hispanic & 9,506 & 22.1 \\
\hline & Other Asian Group & 1,196 & 2.8 \\
\hline & Other Race & 4,602 & 10.7 \\
\hline & Pacific Islanders/Native Hawaiian & 134 & 0.3 \\
\hline & Vietnamese & 930 & 2.2 \\
\hline & White & 30,974 & 72.1 \\
\hline
\end{tabular}

adult questionnaire was used. Section A consists of demographic variables, Section B consists of Health conditions and Section F consists of mental health questions. The described sections' was used to analyze the relationship between depression and diabetes in African American women. The 2011-2012 CHIS surveyed 42,935 adults, 2,799 adolescents, 7,334 children, and more than 44,500 households (California Health Interview Survey, 2012). The survey contains minority populations of Latinos, Asians, and African Americans.

\section{FINDINGS}

The Statistical Package for social Sciences (SPSS) 21 was utilized for these results. The current study was cross sectional in nature and conducted to test independent variables in relation to a dependent variable. The population is self-reported African American women. To test the hypotheses, the chi-square test was used. To be considered significant, the level was set at $p<.05$. For analysis, the dependent variable is diabetes and independent variables are depression, exercise, self-reported race, and gender.

\section{Adult Survey Participants Demographics}

A summary of the participants are presented in Table 1, and there were 42,935 valid responses from participants. Based on the definition of race in the 2000 census, there are seven race categories. Respondents included the following: 2,102 African Americans, 790 American Indian/Alaska Native, 4,302 Asians, 82 Pacific Islander, 30,110 Whites, 4,585 Other single race, and 964 Two or More Races. The 2000 census race question does not mention Latino. Of the total 42,935 respondents there were 2,102 African Americans. African American women are the racial and gender group analyzed for the purpose of this study. The race sample size differs between Table 1 (2000 census definition of race) and Table 2 (self-reported race). In the CHIS 2011-2012 survey, there are 11 self-reported race categories and seven, 2000 census race census categories. For the purposes of this study, the self-reported race of African American was used to validate the link between diabetes (dependent variable) and depression (independent variable).

Using data collected from CHIS 2011-2012, demographics of adult survey participants are presented in Table 3. The questionnaire used only includes adults age 18 or older. In terms of gender, female participation (25,087 or $58.4 \%$ ) exceeded male participation $(17,848$ or $41.6 \%$ ) strongly; that is, a $16.8 \%$ difference in participation by 
Table 3. Diabetes and Depression in Self-Reported Adult African American Women

\begin{tabular}{|c|c|c|c|c|}
\hline \multirow{2}{*}{\multicolumn{2}{|c|}{ Has a doctor ever told you that you have diabetes? }} & \multicolumn{3}{|c|}{$N=1,403(100 \%)$} \\
\hline & & Yes $(\%)$ & No $(\%)$ & Borderline or Pre-diabetes (\%) \\
\hline \multirow{6}{*}{ Levels of Depression } & All of the Time & 16.7 & 72.2 & 11.1 \\
\hline & Most of the Time & 3.4 & 93.1 & 3.4 \\
\hline & Some of the Time & 20.8 & 78.3 & .9 \\
\hline & A Little of the Time & 15.3 & 82.5 & 2.2 \\
\hline & Not at All & 14.5 & 83.7 & 1.8 \\
\hline & Proxy Skipped & 37.5 & 50 & 12.5 \\
\hline
\end{tabular}

female gender existed in the survey and more than half the population sampled was female. Marital status varied within the population sampled, too. Of those sampled, $49.8 \%$ or 21,361 reported being married, 2,260 or 5.3\% reported living with a partner, 11,848 or $27.6 \%$ reported status of widow, separated, or divorced, and 7,466 or $17.4 \%$ reported never being married.

\section{Hypothesis Testing}

In the first hypothesis, an attempt was made to analyze if depression increases the chance of developing diabetes. In the CHIS 2011-2012 survey, the question "has a doctor ever told you that you have diabetes" was asked to adults 18 years or older; the CHIS Adult survey by definition restricts the age limit of respondents to 18 years or older. Using IBM SPSS version 21, cross-tabulation testing was used to analyze the independent and dependent variable. The results of the cross-tabulations were used to generate the percentage by level of depression and the number of people who answered "has a doctor ever told you that you have diabetes?" For purposes of analysis, "has a doctor ever told you that you have diabetes" is the dependent variable, and "feel depressed past 30 days" is the independent variable. The hypothesis "being an African American woman with depression increases the risk of diabetes" was analyzed.

\section{Depression}

There were a total of 1,403 African American women whose responses were analyzed for the first hypothesis. There were a total of 207 African Americans who from the "felt depressed within the last 30 days" who also answered yes to the "has a doctor ever told you that you have diabetes." Three respondents were skipped in this category. There were 1,161 respondents who answered no to both questions about diabetes and depression; four of the respondents were skipped in this category. For the "borderline or pre-diabetes" category a total of 27 respondents reported depression; one respondent was skipped. For those who answered yes to "has a doctor ever told you that you have diabetes" the category percentages are as follows: for "all of the time" $16.7 \%$, "most of the time" 3.4\%, "some of the time" 20.8\%, "a little of the time" 15.3\%, "not at all" 14.5\%, and "proxy skipped" $37.5 \%$. For those who answered no to "has a doctor ever told you that you have diabetes" the category percentages are as follows: for "all of the time" $72.2 \%$, "most of the time" $93.1 \%$, "some of the time" $78.3 \%$, "a little of the time" $82.5 \%$, "not at all" 83.7\%, and "proxy skipped" 50\%. For those who answered borderline or pre-diabetes to "has a doctor ever told you that you have diabetes" the category percentages are as follows: for "all of the time" $11.1 \%$, "most of the time" 3.4\%, "some of the time" .9\%, "a little of the time" $2.2 \%$, "not at all" $1.8 \%$, and "proxy skipped" $12.5 \%$. The data is presented in percentages in Table 3.

\section{Exercise}

In the second hypothesis, an attempt was made to analyze if exercise decreases the chance of developing diabetes in African American women versus the group who responded no. A cross-tabulation testing was used to analyze the independent and dependent variable. The results of the cross-tabulations were used to generate the percentage who exercised versus those who did not and the number of people who answered "has a doctor ever told you that you have diabetes?" For purposes of analysis, "has a doctor ever told you that you have diabetes" is the dependent variable, and "walked at least $10 \mathrm{~min}$ for leisure past 7 days" is the independent variable. The hypothesis "exercise decreases chances of having diabetes in African American women" was analyzed.

Using SPSS 21 cross-tabulation, the data was used for analysis of the question "has a doctor ever told you that you have diabetes" and "walked at least $10 \mathrm{~min}$ for leisure past 7 days." The independent variable will be referred to as "exercise for leisure" for brevity. A total of 1,403 respondents were used as the subset of analysis. Of those who answered yes to "exercise for leisure" and the response to "has a doctor ever told you that you had diabetes" was as follows: for yes $12.3 \%$, no $85.6 \%$, and $2.1 \%$ borderline or pre-diabetes. Of those who answered no to "exercise for leisure" and the response to "has a doctor ever told you that you had diabetes" was as follows: for yes $18.3 \%$, no $79.7 \%$, and $1.9 \%$ borderline or pre-diabetes. Of those who answered in applicable to "exercise for leisure" and the response to "has a doctor ever told you that you had diabetes" was as follows: for yes $10 \%$, no 90\%, and $0 \%$ borderline or pre-diabetes. The data about exercise is presented in Table 4. 
Table 4. Diabetes and Exercise in Self-Reported Adult African American Women

\begin{tabular}{clccc}
\hline \multirow{2}{*}{ Has a doctor ever told you that you have diabetes? } & \multicolumn{2}{c}{$\boldsymbol{N = 1 , 4 0 3 ( \mathbf { 1 0 0 } \% )}$} \\
\cline { 2 - 5 } Exercise for Leisure & Yes & Yes (\%) & No (\%) & Borderline or Pre-diabetes (\%) \\
\cline { 2 - 5 } & No & 12.3 & 85.6 & 79.7 \\
\cline { 2 - 5 } & Inapplicable & 18.3 & 90 & 1.9 \\
\hline
\end{tabular}

\section{Chi-square Testing}

For the first hypothesis, there were a total of 1,403 self-reported adult African American women. The population under study was asked "has a doctor ever told you that you have diabetes?" Responses included 210, who answered yes, 1165 who answered no, and 28 answered borderlines or pre-diabetes. A chi-square test was performed to analyze the relationship between levels of depression and diabetes. From the test results, there was an association between levels of depression and diabetes. The Pearson chi-squared value of 22.918, $d f$ of 10, and $p=.011$ is statistically significant. Analysis supports hypothesis one that there is a significant relationship between level of depression and diabetes. The null hypothesis was rejected.

For the second hypothesis, there were also a total of 1,403 self-reported adult African American women. The second hypothesis proposes that African Americans who exercise have less risk in acquiring diabetes than those who do not exercise. A Pearson chi-square test was conducted to analyze the relationship between exercise and diabetes. The results of the chi-square test suggest a significant relationship between the variables. The Pearson chi-square value of $10.408, d f$ of 4 , and $p=.034$ is a statistically significant relationship. Results of the analysis support the second hypothesis.

\section{CONCLUSION, LIMITATIONS AND RECOMMENDATIONS}

The objective of this research was to analyze the disproportionate share of diabetes amongst African American women and to investigate the reasons it is more common in this population. This study focused on African American women in the state of California using CHIS 2011-2012 survey data. Taken from the CHIS 2011-2012 survey, the question "has a doctor ever told you that you have diabetes" served as the dependent variable and the independent variables used were self-reported African American, female gender, depression, and exercise. The independent variable African American and female were used to select the population of interest as a subset of the data. The two independent variables, depression and exercise, were analyzed to suggest that these variables were the reason diabetes is common among African American women.

For the purpose of the study, African American women were identified using the variable labeled "SelfReported African American" and by respondents identifying themselves as female in the variable labeled "Gender." There was more self-reported African American than those classified according to the 2000 census definition of race. Because of the difference, the self-reported African American variable was used instead of the 2000 census definition. The subset of self-reported African American female was used to perform the two independent variables of depression and exercise for hypothesis testing.

Diabetes and depression are usually comorbid illnesses. Directional effect can be questioned. Does depression cause diabetes or does diabetes cause depression? Evidence exists that depression has at minimum been linked to worsened outcomes for those with diabetes. Diabetics have a difficult enough time with managing diet, weight, exercise, blood glucose monitoring, and adherence to medication regimens. With such a burden depression can exacerbate non-adherence to medication regimens and cause a spiraling downward effect. Depression, if detected early, could save the healthcare system millions of dollars. Depression has been described as the common cold of mental health, and the rates of depression around the world appear to be worsening (Marcus et al., 2012). More research needs to be conducted to determine the influence depression has on developing diabetes.

Reasons for the disparity in healthcare treatment range from patient mistrust of the system to lack of cultural competence. Factors affecting the level of care administered include affordability of health insurance, cost of copayments, knowledge of the health system, and distrust of physicians. Physicians affect the outcomes of patients through biases and financial incentives to over treat patients that result in cynicism towards physicians. Health plans can influence patient outcomes by charging out-of-pocket expenses, geographical proximity, and the staff cultural competence. At the community level, factors include access to community health workers and the availability of state administered Medicaid.

After selecting African American women, the population under study, cross-tabulation was used for the dependent variable (shortened in name to) diabetes and run against each independent variable to test for significance. Instead of using the 2000 census definition of race, the self-reported African American variable was used to select the subset of the population. The subset of data selected suggests a link between diabetes and 
depression, and the data suggests a link between exercise and diabetes, too. Because the data was restricted to the state of California more research needs to be conducted to determine the extent of the independent variables' influence on diabetes.

\section{Limitations of the Study}

The amount of data on African American women was smaller than desired because of the specificity of this study. Other parts of the United States contain larger population sizes of African Americans where diabetes is a prevalent condition. The sample size appears large but those reporting depression and diabetes totaled 207 respondents out of a total of 1,403 adult African American women. Another limitation on the study is the aggregation of Type 1 and Type 2 diabetes together. As one group of researchers in the literature discussed, Type 1 and Type 2 diabetes have different etiologies (Van Tilburg et al., 2001); yet most research makes no distinctions between the two. The effects of depression and exercise may have varied with the Type of diabetes. Within the relatively small population diabetes was shown to have a relationship with depression and exercise. A major obstacle to measuring levels of depression within the African American community is breaking a barrier of mistrust. Because of historical racism, many African Americans distrust the health care system and instead feel the need to work with someone of a similar ethnic and experiential background.

According to Cummings et al. (2013), the South Eastern U.S. has the largest numbers of African Americans and the highest rates of Type 2 diabetes. The CHIS 2011-2012 survey did not capture a desired, larger sample of African Americans. The population sampled from CHIS included 1,403 adult African American women. A larger sample size might have increased the statistical significance for both depression and exercise as factors. Despite the sample size, a statistically significant relationship existed between each of the independent variables, exercise and depression, and the dependent variable diabetes.

\section{Recommendations for Future Study}

With an increasing percentage of African Americans diagnosed as diabetic, the negative consequences for the population will increase. Diabetes has life threatening consequences ranging from blindness to loss of limbs. Diabetes and depression are often comorbid. The direction of causation between diabetes and depression may be bidirectional. More research needs to be conducted to strengthen the finding that depression causes diabetes. Depression and diabetes work in a negative, synergistic way to spiral a patient down; depression leads to medication non-adherence, lack of regimen follow through, and neglect of basic self-care.

Screening of depression at an early age could help prevent the onset of pre-diabetes and prevent the onset of full diabetes. A reliable and valid test for depression such as the PHQ 2/9 could be administered as part of a physical or made a standard practice in primary care. People currently diagnosed with diabetes in particular would be screened for depression. Cohort studies may be needed to determine the strength of a causal relationship between depression and diabetes. Also, clinical interviews strengthen a study in determining a diagnosis of depression. Most studies use a well-respected depression inventory that tests for symptoms and cannot make an actual diagnosis of clinical depression. Current studies rely on cross-sectional data making it more difficult to assess a causal relationship between diabetes and depression.

Studies have focused on various parts of the south eastern United States where African Americans have a high population density and high prevalence of diabetes. Future studies should aim to investigate a broader sample of African Americans in the other regions of the United States. African Americans face issues of cultural competence by physicians and a distrust of a health system perceived to be for whites. Physicians need to be trained in culturally competent care to help African Americans who have a distrust gain better access to care. In particular, the training of more African American community health workers and physicians would bridge the gap between those who need access but are unwilling to receive the care they need.

\section{REFERENCES}

American Diabetes Association. (2013a). Data from the 2011 national diabetes fact sheet. [online] Available at: http://www.diabetes.org/diabetes-basics/diabetes-statistics/

American Diabetes Association. (2013b). Living with diabetes: African americans and complications. [online] Available at: http://www.diabetes.org/living-with-diabetes/complications/african-americans-andcomplications.html

Black, S.A. (2002). Diabetes, diversity, and disparity: What do we do with the evidence? American Journal of Public Health, 92(4), pp. 543-548. https://doi.org/10.2105/AJPH.92.4.543

Centers for Disease Control. (2011). National diabetes fact sheet, 2011. [online] Available at: http://www.cdc.gov/diabetes/pubs/pdf/ndfs_2011.pdf 
California Health Interview Survey. (2012). CHIS 2011-2012 adult questionnaire (Version 10.1) [Data file]. Los Angeles, CA: UCLA Center for Health Policy Research.

Cummings, D.M., Lutes, L.D., Littlewood, K., DiNatale, E., Hambidge, B. and Schulman, K. (2013). EMPOWER: A randomized trial using community health workers to deliver a lifestyle intervention program in african american women with type 2 diabetes: Design, rationale, and baseline characteristics. Contemporary Clinical Trials, 36(1), pp. 147-153. https://doi.org/10.1016/j.cct.2013.06.006

Katon, W.J., Young, B.A., Russo, J., Lin, E.H., Ciechanowski, P., Ludman, E.J. and Von Korff, M.R. (2013). Association of depression with increased risk of severe hypoglycemic episodes in patients with diabetes. The Annals of Family Medicine, 11(3), pp. 245-250. https://doi.org/10.1370/afm.1501

Marcus, M., Yasamy, M.T., van Ommeren, M., Chisholm, D. and Saxena, S. (2012). Depression a global public health concern. [online] Available at: http://www.who.int/mental_health/management/depression/who_paper_depression_wfmh_2012.pdf

Miller, S.T., Marolen, K.N. and Beech, B.M. (2010). Perceptions of physical activity and motivational interviewing among rural african-american women with type 2 diabetes. Women's Health Issues, 20(1), pp. 43-49. https://doi.org/10.1016/j.whi.2009.09.004

Murrock, C.J., Taylor, E. and Marino, D. (2013). Dietary challenges of managing type 2 diabetes in AfricanAmerican women. Women \& Health, 53(2), pp. 173-184. https:// doi.org/10.1080/03630242.2012.753979

Van Tilburg, M.A., McCaskill, C.C., Lane, J.D., Edwards, C.L., Bethel, A., Feinglos, M.N. and Surwit, R.S. (2001). Depressed mood is a factor in glycemic control in type 1 diabetes. Psychosomatic Medicine, 63(4), pp. 551-555. https://doi.org/10.1097/00006842-200107000-00005

Wild, S., Roglic, G., Green, A., Sicree, R. and King, H. (2004). Global prevalence of diabetes estimates for the year 2000 and projections for 2030. Diabetes Care, 27(5), pp. 1047-1053. https://doi.org/10.2337/diacare.27.5.1047

Ylitalo, K.R., Karvonen-Gutierrez, C.A., Fitzgerald, N., Zheng, T.H., Sternfeld, B., El Khoudary, S.R. and Harlow, S.D. (2013). Relationship of race-ethnicity, body mass index, and economic strain with longitudinal self-report of physical functioning: The study of women's health across the nation. Annals of Epidemiology, 23(7), pp. 401408. https://doi.org/10.1016/j.annepidem.2013.04.008 